\title{
Association of Genetic Variants in TPMT, ITPA, NUDT15 With Azathioprine-Induced Myelosuppression in Southwest China Patients With Autoimmune Hepatitis
}

\author{
Qiang Miao \\ West China Hospital of Sichuan University \\ Lin Yan \\ West China Hospital of Sichuan University \\ Yanhong Zhou \\ West China Hospital of Sichuan University \\ Yi Li \\ West China Hospital of Sichuan University \\ Yuangao Zou \\ West China Hospital of Sichuan University \\ Lanlan Wang \\ West China Hospital of Sichuan University \\ Yangjuan Bai \\ West China Hospital of Sichuan University \\ Junlong Zhang ( $\square$ immune_hx@126.com) \\ West China Hospital of Sichuan University
}

\section{Research Article}

Keywords: Azathioprine, Myelosuppression, NUDT15, TPMT, ITPA, Autoimmune hepatitis

Posted Date: December 11th, 2020

DOI: https://doi.org/10.21203/rs.3.rs-123603/v1

License: (9) (1) This work is licensed under a Creative Commons Attribution 4.0 International License. Read Full License

Version of Record: A version of this preprint was published at Scientific Reports on April 12th, 2021. See the published version at https://doi.org/10.1038/s41598-021-87095-0. 


\section{Abstract}

Aims: To investigate the influence of TPMT*3C, ITPA, NUDT15, and 6-thioguanine nucleotides (6-TGN) on AZA-induced myelosuppression in Southwest China AlH patients.

Methods: A total of 113 Chinese AlH patients with AZA maintenance treatment were evaluated. Collect the relevant clinical data of patients from the hospital information system. TPMT*3C(rs1142345), ITPA(rs1127354) and NUDT15(rs116855232) genotyping was detected by TaqMan double fluorescent probe. The concentration of 6-TGN was determined using UPLC-MS/MS method.

Results: 40 (35.4\%) patients had different degrees of myelosuppression. The NUDT15 variant was associated with leukopenia $\left(P=8.26 \times 10^{-7}\right.$; $\mathrm{OR}=7.5 ; 95 \% \mathrm{Cl}, 3.08-18.3)$ and neutropenia $\left(P=3.54 \times 10^{-6} ; \mathrm{OR}=8.05 ; 95 \% \mathrm{Cl}, 2.96-21.9\right)$. However, no significant association was observed for TPMT*3C and ITPA variants ( $P>0.05$ ). There was no significant difference of 6-TGN concentration between patients with or without myelosuppression $(P=0.556)$, and no association was found in patients with TPMT*3C, ITPA, NUDT15 variants alleles $(P>0.05)$. However, we found that the body mass index may affect the corrected 6-TGN level $(P=0.026)$.

Conclusion: Our study once again confirmed that NUDT15 variants are a potential independent risk predictor for AZA-induced leukopenia and neutropenia. Also, we found that the detection of 6-TGN concentration in red blood cells does not reflect AZA treatment's efficacy and toxicity. New biomarkers for AZA therapeutic drug monitoring need further research to explore.

\section{Introduction}

Azathioprine (AZA) is a prodrug of thiopurine and has been used as a classic immunosuppressant in the clinical treatment of autoimmune diseases for more than 60 years. Autoimmune hepatitis (AIH) guidelines issued by the European Hepatology Society in 2015 proposed the combination therapy of prednisone and AZA as the first-line program for induction of remission and maintenance therapy for AlH patients. ${ }^{1}$ However, the AZA medication varies significantly between individuals, and about $15 \%$ of patients have adverse drug reactions leading to treatment interruption. ${ }^{2}$ Among them, the most common and severe adverse reaction in the early stage of treatment is myelosuppression. Patients are usually asymptomatic but significantly increase the risk of life-threatening infections. ${ }^{3}$

The individual difference of AZA treatment is closely related to its metabolism in the body. As a prodrug, AZA has no biological activity. 6thioguanine nucleotides (6-TGN) and methylation products 6-Methylmercaptopurine ribonucleotides (6-MMPr $)^{4,5}$ are the final active metabolites, which cause the risk of myelosuppression and liver toxicity. Its metabolic process is complex, with various enzymes involved and affected by enzyme gene polymorphisms. Thiopurine methyltransferase (TPMT) is a crucial enzyme in the metabolism of AZA. The TPMT gene mutation leads to the decrease or deletion of TPMT activity, which affects the balance between the active metabolites 6-MMPr and 6-TGN. Patients with gene mutations or low enzymatic activity tend to increase the concentration of 6-TGN, which is prone to myelosuppression. ${ }^{6}$ However, the risk allele frequency of $T P M T \star 3 C T>C$ is low in East Asian (1.3\%) populations. ${ }^{7}$ Therefore, the TPMT genotype testing and mercaptopurine dosage guidelines issued in European and American countries do not apply to Asian and Chinese populations. It cannot fully explain the low tolerable dose of AZA in Asian population and the high incidence of adverse reactions.

Inosine triphosphate pyrophosphatase (ITPA) is widely present in various organs and tissues, including red blood cells. Its role is to catalyze inosine triphosphate (ITP) hydrolysis into Inosine monophosphate (IMP) and protect cells from the accumulated nucleotides' damage. This cyclic reaction still exists in the process of AZA metabolism. The incidence of ITPA 94C $>$ A mutation in Asian population is as high as $14 \%-19 \% .{ }^{8}$ Studies have shown that ITPA genotype can explain and predict the resistance and side effects of thiopurines therapy and change treatment outcomes. ${ }^{9,10}$ In 2014, a study on thiopurines-related leukopenia in patients with inflammatory bowel disease (IBD) found that NUDT15 c.415C > T gene mutation is closely related to this. ${ }^{11}$ Subsequent reports in Japan, China and India ${ }^{12-15}$ all found that NUDT15 c. $415 C>T$ gene polymorphism is closely related to AZA-induced leukopenia. In patients with acute lymphoblastic leukemia, especially in Asian population, it has also been found that NUDT15 C.415C > T gene mutations may be related to thiopurines tolerance and myelosuppression. ${ }^{16,17}$ The application of AZA in other autoimmune diseases, especially in AlH, is more widely used than IBD. However, only two related reports have been found for AlH patients, including a case report. ${ }^{18,19}$ There has not been a comprehensive assessment of the relationship between genetic variants of TPMT*3CT $>C$, ITPA $94 C>A, N U D T 15 C .415 C>T$ and AZA toxicity in southwest China AlH patients.

The primary purpose of our study is to investigate the relationship between TPMT*3C T $>C$, ITPA 94C $>A$ and NUDT15 C.415C $>T$ single nucleotide variants and AZA-induced myelosuppression in AlH patients. Besides, we tried to explore the influence of 6-TGN levels in red blood cells on myelosuppression in patients with AlH to clarify the value of thiopurines metabolite detection in guiding drug dose adjustment in the treatment of such patients. 


\section{Materials And Methods}

\subsection{Subjects}

In this study, a total of 113 patients with AlH who received AZA maintenance treatment from September 2017 to September 2019 in West China Hospital of Sichuan University were included. The inclusion criteria included patients who were clinically diagnosed as AlH and received AZA treatment for more than 12 weeks, were followed up regularly in our hospital and were 18 years or older. Exclusion criteria include patients under the age of 18 , or patients with a recent history of blood transfusion and other medications that may lead to myelosuppression, or patients during pregnancy and lactation, or patients with an incomplete medical history and not regularly followed up. Collect the relevant clinical data of patients from the hospital information system (HIS), including gender, age, height, weight, the dosage of medication, and regular follow-up to monitor the results of routine blood tests. This study was performed following the Declaration of Helsinki and approved by the Ethics Committee of West China Hospital of Sichuan University. All enrolled patients were provided written informed consent.

\subsection{Treatment and Toxicity}

According to body weight, the instructions administer the initial dose of AZA treatment for AlH patients, usually 1.0-1.5 mg/kg per day. Complete blood cell count (CBC) was performed weekly for the first month after treatment beginning and every two weeks for the next two months. After three months of treatment or at the time point when AZA toxicity occurred for 6-TGN concentration and genetic testing, collect blood samples from patients. The primary time endpoint of follow-up was 12 weeks, and the secondary time endpoint was the occurrence of myelosuppression and withdrawal or switching to other drugs. Regarding reducing the drug dose and the drug's discontinuation, the clinician responsible for the treatment decides.

According to the World Health Organization standards for acute and subacute toxicity of anticancer drugs, myelosuppression is defined as white blood cell (WBC) count less than $4 \times 10^{9} / \mathrm{L}$, or platelet (PLT)count less than $100 \times 10^{9} / \mathrm{L}$, or neutrophil (NEU) count less than $2 \times 10^{9} / \mathrm{L}$. The relevant hematological indicators gradually decreased during the treatment monitoring period for patients with mild myelosuppression before treatment. After a comprehensive assessment by the clinician in charge of treatment, when excluding other diseases that cause myelosuppression, it is considered to be AZA-induced myelosuppression.

\subsection{Gene Analysis}

Total genomic DNA was extracted from peripheral blood using the YAOJINBAO $\rightarrow$ DNA purification Kit (Beijing Sino-Era Gene Tech Co. Ltd, China) according to the manufacturer's instructions. TPMT*3C T > C(rs1142345), ITPA 94C >A(rs1127354) and NUDT15 C.415C > T (rs 116855232) genotyping was detected by allele-specific polymerase chain reaction (PCR) combined with TaqMan double fluorescent

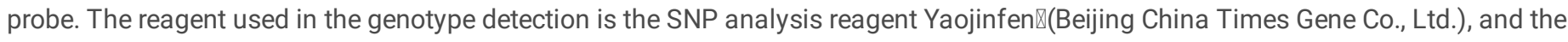
detection instrument is Fluotec 48E Trace fluorescence detector (Xi'an TianLong Science and Technology Co. Ltd). The reaction system temperature for TPMT*3C, ITPA and NUDT 15 was $58{ }^{\circ} \mathrm{C}, 64^{\circ} \mathrm{C}$ and $60{ }^{\circ} \mathrm{C}$, respectively. The standard test procedure was 55 cycles, and the total reaction time was generally within $2.5 \mathrm{~h}$. Both negative and positive controls were included in all sample analysis process to ensure the authenticity of the results.

\subsection{6-TGN concentration determination}

The concentration of 6-TGN, an active metabolite of azathioprine, was determined using our previously published ultra-performance liquid chromatography-tandem mass spectrometry (UPLC-MS/MS) method. ${ }^{20}$ The result report is similar to the previous study, expressed in $\mathrm{pmol} / 8 \times 10^{8} \mathrm{RBCs}$.

\subsection{Statistical Analysis}

Statistical analysis was performed using IBM SPSS software version 23.0 (SPSS Inc., Chicago, IL, USA). Hardy Weinberg equilibrium (HWE) was calculated for each polymorphism studied. $P>0.05$ (Chi-squared statistics) was considered to indicate equilibrium. Continuous data were summarized using medians and interquartile ranges (IQRs) and were compared using the Kruskal-Wallis H-test or Mann-Whitney U-test. Categorical variables are reported as frequencies and percentages, and Pearson Chi-square tests or Fisher's exact tests were performed to analyze differences between two independent groups. The odds ratios (OR) and 95\% confidence interval of the allele model were determined by logistic regression analysis. All statistical tests were 2-tailed and a $P<0.05$ was deemed significant.

\section{Results}

\subsection{Patient characteristics}


According to the inclusion and exclusion criteria, 113 eligible patients were included in the study. Most of them were female patients $(n=97$, $85.8 \%$ ), and the ratio of female to male was about 6:1. The age ranges from 26 to 77 years old. These patients' characteristics were summarized in Table 1. In the end, 40 (35.4\%) patients had different degrees of myelosuppression. Age, gender, weight, smoke, the initial dose of AZA, liver function indicators, baseline WBC count, PLT count, and NEU count were not significantly different between individuals with or without myelosuppression $(P>0.05)$. Patients with myelosuppression have a lower height than those without myelosuppression (1.56 $\mathrm{m}$ vs. $1.60 \mathrm{~m}, P=0.018$ ). There were also significant differences in the distribution of body mass index (BMI) between the two $\operatorname{groups}(P=0.003)$. The proportion of patients with a BMI of less than $18.5 \mathrm{~kg} / \mathrm{m}^{2}$ in the myelosuppression group was $15 \%$, while the proportion of patients in the without myelosuppression group was $0 \%$ (Table 1 ).

Table 1

The baseline characteristics of included subjects in this study [Median(IQR)]

\begin{tabular}{|c|c|c|c|}
\hline Clinical features & $\begin{array}{l}\text { With myelosuppression } \\
(\mathrm{N}=40)\end{array}$ & $\begin{array}{l}\text { Without myelosuppression } \\
(\mathrm{N}=73)\end{array}$ & $P$ \\
\hline Age (years) & $52.0(45.2,60.8)$ & $50.0(43.0,57.0)$ & 0.168 \\
\hline Female/male & $36 / 4$ & $61 / 12$ & 0.348 \\
\hline Height (m) & $1.56(1.52,1.60)$ & $1.60(1.55,1.62)$ & $0.018^{* *}$ \\
\hline Weight (kg) & $56.0(50.5,63.0)$ & $59.0(52.8,65.0)$ & 0.127 \\
\hline $\mathrm{BMI}\left(\mathrm{kg} / \mathrm{m}^{2}\right)$ & $23.3(20.6,24.9)$ & $23.1(21.4,24.7)$ & 0.714 \\
\hline$<18.5$ & $15.0 \%(6 / 40)$ & $0 \%(0 / 73)$ & $0.003^{* *}$ \\
\hline $18.5 \leq \mathrm{BMI} \leq 24$ & $52.5 \%(21 / 40)$ & $63.0 \%(46 / 73)$ & \\
\hline$>24$ & $32.5 \%(13 / 40)$ & $37.0 \%(27 / 73)$ & \\
\hline AZA dose $\left(\mathrm{mg} \cdot \mathrm{kg}^{-1} \cdot \mathrm{d}^{-1}\right)$ & $1.07(0.85,1.27)$ & $0.98(0.86,1.44)$ & 0.978 \\
\hline WBC_0 $0^{\dagger}\left(10^{9} / \mathrm{L}\right)$ & $6.55(5.24,7.91)$ & $6.53(5.29,7.52)$ & 0.570 \\
\hline PLT_0 $0^{\dagger}\left(10^{9} / \mathrm{L}\right)$ & $274(183,344)$ & $238(176,338)$ & 0.583 \\
\hline NEU_0 ${ }^{\dagger}\left(10^{9} / \mathrm{L}\right)$ & $3.25(2.71,4.24)$ & $3.40(2.79,4.35)$ & 0.606 \\
\hline $\operatorname{ALT}(\mathrm{IU} / \mathrm{L})$ & $122.5(82.5,147.8)$ & $120.8(77.7,152.6)$ & 0.881 \\
\hline AST(IU/L) & $128.4(97.6,154.7)$ & $127.3(99.1,168.4)$ & 0.652 \\
\hline ALP(IU/L) & $172.6(120.6,222.4)$ & $187.9(140.2,214.5)$ & 0.631 \\
\hline GGT(IU/L) & $180.3(120.2,236.5)$ & $166.4(81.1,248.3)$ & 0.517 \\
\hline$T P M T * 3 C T T / T C / C C$ & $40 / 0 / 0$ & $71 / 2 / 0$ & 0.539 \\
\hline ITPA $94 C>A C C / C A / A A$ & $27 / 13 / 0$ & $50 / 22 / 1$ & 0.741 \\
\hline NUDT15 c.415C > T CC/CT/TT & $26 / 11 / 3$ & $62 / 11 / 0$ & $0.012^{\star \star}$ \\
\hline Smoke Yes/No & $0 / 40$ & $5 / 68$ & 0.159 \\
\hline
\end{tabular}

Abbreviations: BMI, body mass index; AZA, azathioprine; WBC, white blood cell count; PLT, platelet count; NEU, neutrophil count; ALT, alanine aminotransferase; AST, aspartate aminotransferase; ALP, alkaline phosphatase; GGT, glutamyl transferase.

${ }^{\dagger}$ Represents the results before starting azathioprine treatment.

**Significant $(P<0.01)$.

TPMT*3C, ITPA and NUDT15 genotype distributions were in Hardy Weinberg equilibrium among the included population $(P=1.00, P=0.53$ and $P=0.822)$. The detailed distribution was shown in Table 2 . No $T P M T * 3 C(T>C)$ homozygote (CC) was detected in the study, 2 cases were heterozygotes (TC, 1.8\%), and the remaining 111 cases were wild-type (TT, 98.2\%), the $\mathrm{C}$ and $\mathrm{T}$ allele frequencies were $0.9 \%$ and $99.1 \%$, respectively. Among the 113 analyzed individuals, 77 patients were ITPA 94C > A wild-type (CC, 68.1\%), 35 patients were heterozygotes (CA, $31.0 \%$ ) and only one subject was homozygote (AA, $0.9 \%$ ), and the frequencies of $\mathrm{C}$ and $\mathrm{A}$ alleles were $83.6 \%$ and $16.4 \%$, respectively. In the same cohort, the numbers of subjects displayed NUDT15 c.415(C > T) genotypes CC, CT, and TT were 88(77.8\%), 22(19.5\%), and 3(2.7\%), 
respectively. The frequency of variant allele T was $12.4 \%$. There were significant differences in the genotype distribution of NUDT15 C.415(C $>T$ ) between the individuals with and without myelosuppression $(P=0.012)$, while no significant differences were observed in the TPMT*3C $(T>C)$ and ITPA 94C $>A$ genotypes $(P<0.05$, Table 1$)$.

Table 2

Allele distribution of NUDT15 c.415C > T, ITPA 94C > A and TPMT*3C genotypes.

\begin{tabular}{|c|c|c|c|c|c|c|}
\hline \multirow[t]{2}{*}{ Gene } & \multirow[t]{2}{*}{ Genotype } & \multirow[t]{2}{*}{$\mathbf{N}$} & \multirow[t]{2}{*}{ Genotype frequency (\%) } & \multicolumn{3}{|c|}{ Allelic association } \\
\hline & & & & Allele & Allele frequency (\%) & HWE $P$-value \\
\hline \multirow[t]{2}{*}{$T P M T \star 3 C T>C(r s 1142345)$} & TT & 111 & 98.2 & $\mathrm{~T}$ & 99.1 & 1.00 \\
\hline & TC & 2 & 1.8 & C & 0.9 & \\
\hline \multirow[t]{3}{*}{ IPTA94C > A (rs1127354) } & $\mathrm{CC}$ & 77 & 68.1 & C & 83.6 & 0.53 \\
\hline & $\mathrm{CA}$ & 35 & 31.0 & $A$ & 16.4 & \\
\hline & AA & 1 & 0.9 & & & \\
\hline \multirow[t]{3}{*}{ NUDT15 c.415C > T (rs116855232) } & $\mathrm{CC}$ & 88 & 77.8 & C & 87.6 & 0.822 \\
\hline & CT & 22 & 19.5 & $\mathrm{~T}$ & 12.4 & \\
\hline & TT & 3 & 2.7 & & & \\
\hline
\end{tabular}

\subsection{Association of phenotype with myelosuppression}

Among the 113 patients, $47(41.6 \%)$ subjects were variant individuals and 66(58.4\%) were wild-type patients. No significant differences were observed in age, BMI, AZA dose, 6-TGN concentration, and Correction 6-TGN concentration between the individuals in the variation and wildtype groups ( $P>0.05$, Table 3 ). We compared myelosuppression indicators at the 12th week or when adverse events occurred between the two groups. It was found that the median levels of WBC_12w and NEU_12w in the variation group were $4.99(4.03,6.66) \times 10^{9} / \mathrm{L}$ and 2.82 $(2.21,4.04) \times 10^{9} / \mathrm{L}$, respectively, while wild-type patients were $6.39(4.64,7.72) \times 10^{9} / \mathrm{L}$ and $3.67(2.75,5.22) \times 10^{9} / \mathrm{L}$, and there were significant differences between the two groups $(P=0.003$ and $P=0.002)$. However, the median level of PLT_12w was not significantly $\operatorname{different}(P>0.05$, Table 3$)$. There were also no significant differences in myelosuppression incidence between variant patients and wild-type patients $(P>0.05$, Table 3$)$.

Table 3

Analysis of related indexes between patients with genetic variation and wild-type patients [Median(IQR)]

\begin{tabular}{|c|c|c|c|}
\hline & Variation group $^{\dagger}(n=47)$ & Wild-type group $(n=66)$ & $P$ \\
\hline Age (years) & $54(45,62)$ & $50(43,54)$ & 0.063 \\
\hline $\mathrm{BMI}\left(\mathrm{kg} / \mathrm{m}^{2}\right)$ & $22.5(20.6,24.2)$ & $23.2(21.7,26.1)$ & 0.229 \\
\hline AZA dose $\left(\mathrm{mg} \cdot \mathrm{kg}^{-1} \cdot \mathrm{d}^{-1}\right)$ & $0.95(0.85,1.18)$ & $1.08(0.87,1.47)$ & 0.083 \\
\hline 6-TGN (pmol/8 × 108 RBCs) & $125.29(87.43,237.21)$ & $115.15(64.16,196.81)$ & 0.262 \\
\hline Correction $6-\mathrm{TGN}^{\ddagger}\left(\mathrm{pmol} / 8 \times 10^{8} \mathrm{RBCs} . \mathrm{mg} . \mathrm{kg} . \mathrm{d}\right)$ & $0.037(0.021,0.066)$ & $0.036(0.019,0.062)$ & 0.958 \\
\hline WBC_12W§ $\left(10^{9} / \mathrm{L}\right)$ & $4.99(4.03,6.66)$ & $6.39(4.64,7.72)$ & $0.003^{* *}$ \\
\hline PLT_12w§ $\left(10^{9} / \mathrm{L}\right)$ & $122(89,220)$ & $140(96,196)$ & 0.942 \\
\hline NEU_12W§ $\left(10^{9} / L\right)$ & $2.82(2.21,4.04)$ & $3.67(2.75,5.22)$ & $0.002 * \star$ \\
\hline Myelosuppression & & & 0.346 \\
\hline Yes & $19(40.4 \%)$ & $21(31.8 \%)$ & \\
\hline No & $28(59.6 \%)$ & $45(68.2 \%)$ & \\
\hline
\end{tabular}

Abbreviations: BMI, body mass index; AZA, azathioprine; 6-TGN, 6-thioguanine nucleotides; WBC, white blood cell count; PLT, platelet count; NEU, neutrophil count; RBC, red blood cell. 
${ }^{\dagger}$ At least one gene polymorphic mutation in TPMT*3C (rs1142345), ITPAC.94C >A (rs1127354) and NUDT15c.415C > T (rs116855232).

‡6-TGN concentration corrected by body weight and daily dose.

$\S$ start azathioprine treatment for 12 weeks or at the time when adverse events occurred.

**Significant $(P<0.01)$.

\subsection{Association of genotypes with myelosuppression}

We further analyzed the relationship between myelosuppression related indicators and TPMT*3C T > C ( s 1142345), ITPA94C >A(rs1127354) and NUDT15c.415C > T (rs116855232) genotypes. The detailed results are shown in Table 4. Of the 111 patients with wild type (TT) of TPMT*3C, 15 (13.5\%) patients occurred leukopenia, 34 (30.6\%) patients had thrombocytopenia, and 10 (9.0\%) patients occurred neutropenia. Two cases of heterozygotes (TC) patients did not develop myelosuppression, but their AZA dosage was less than 1 mg $\cdot \mathrm{kg}^{-1} \cdot \mathrm{d}^{-}$ 1. There was no significant correlation between AZA-induced leukopenia, thrombocytopenia, neutropenia and $T P M T * 3 C$ genotypes $(P=$ $0.747,0.351$ and 0.658 , respectively). In 77 patients with wild-type (CC) of ITPA 94C >A, the numbers of leucopenia, thrombocytopenia, neutropenia were 7 (9.1\%), 24 (31.2\%) and 3 (8.6\%), respectively. Among the 35 heterozygous (CA) patients, 8 (22.9\%) patients had leukopenia, 10 (28.6\%) patients occurred thrombocytopenia, and 3 (8.6\%) patients occurred neutropenia. Only one patient was homozygous (AA). Similarly, this patient did not develop myelosuppression, and his AZA dose was also less than $1 \mathrm{mg} \cdot \mathrm{kg}^{-1} \cdot \mathrm{d}^{-1}$. Compared with wild-type (CC), patients with variant allele $\mathrm{A}(\mathrm{CA}+\mathrm{AA})$ have no significant difference in the risk of leukopenia, thrombocytopenia, and neutropenia $(P=$ $0.102,0.657$ and 0.862 , respectively). 
Table 4

Association of myelosuppression with NUDT15 c.415C > T, ITPA 94C > A and TPMT*3C genotypes

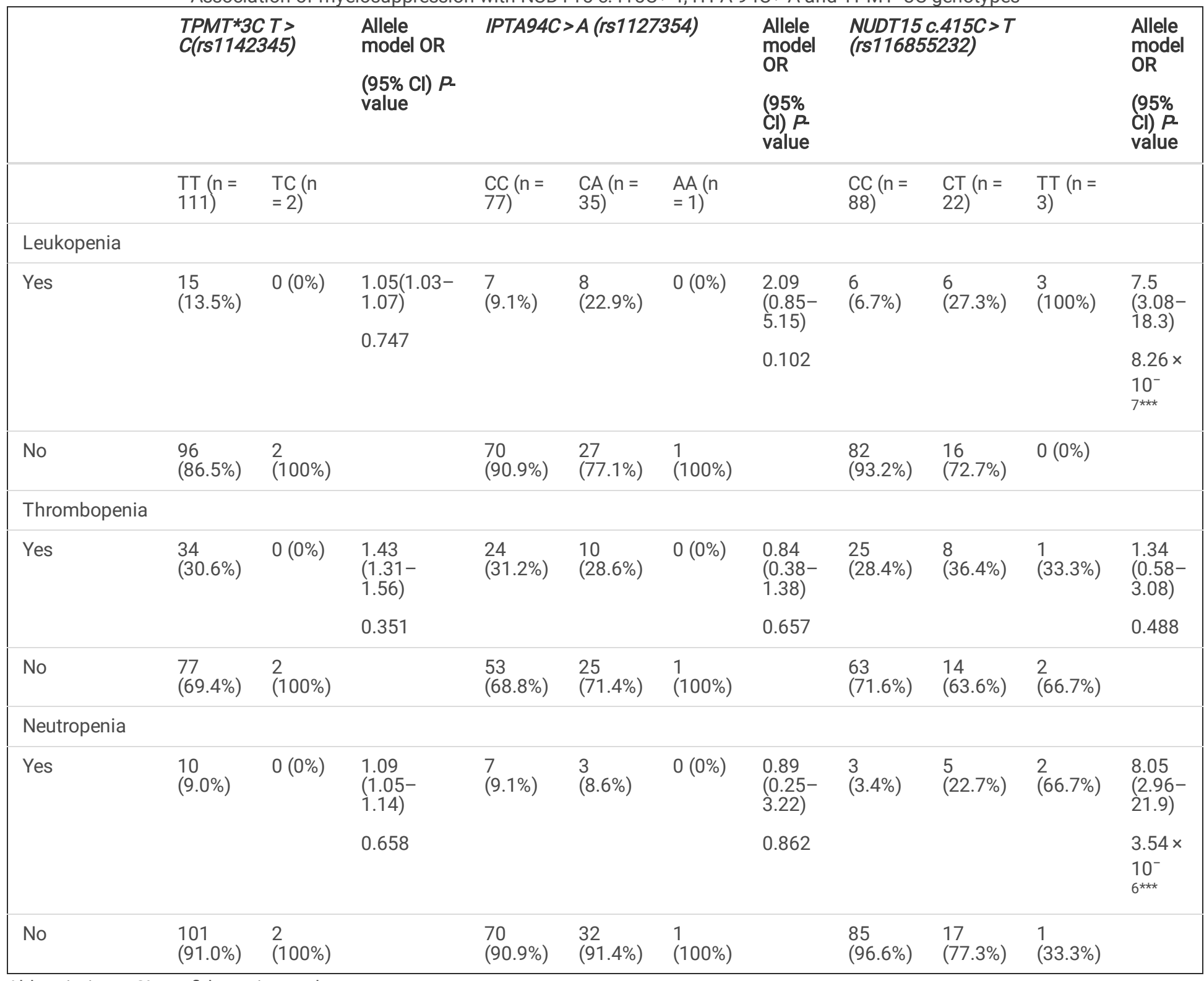

Abbreviations: $\mathrm{Cl}$, confidence interval.

$\star \star \star$ Significant $(P<0.001)$.

When evaluated by NUDT15c.415C>T genotype, AZA-induced leukopenia was observed in 6 (6.7\%) patients with the CC allele and 6 (27.3\%) patients with the CT allele. $3(100 \%)$ patients with homozygote allele (TT) all suffered leukopenia. Leukopenia was significantly associated with the NUDT15c.415C>T variant allele T $\left(P=8.26 \times 10^{-7} ; \mathrm{OR}=7.5 ; 95 \% \mathrm{Cl}, 3.08-18.3 ;\right.$ Table 4$)$. The incidence of neutropenia in patients with CC, CT and TT genotypes was 3.4\% (3/88), 22.7\% (5/22), and 66.7\% (2/3), respectively. Compared with the wild-type genotype (CC), patients carrying variant allele T $(\mathrm{CT}+\mathrm{TT})$ had a much higher risk of developing neutropenia $\left(P=3.54 \times 10^{-6}\right.$; OR = 8.05; 95\% $\mathrm{Cl}, 2.96-21.9$; Table 4). The NUDT15 variant allele T had high predictability for leukopenia and neutropenia ( $36 \%$ and $28 \%$ ). However, there is no significant difference in thrombocytopenia incidence among patients with different genotypes $(P=0.488)$. 2(66.7\%) of the homozygous (TT) patients' AZA dosage was less than $1 \mathrm{mg} \cdot \mathrm{kg}^{-1} \cdot \mathrm{d}^{-1}$.

3.4 Relationship between 6-TGN concentration and myelosuppression

In 113 patients, the concentration of 6-TGN ranged from 2.07 to $2554.09 \mathrm{pmol} / 8 \times 10^{8} \mathrm{RBCs}$, and the median (interquartile range) concentration was $123.34(79.89,231.77) \mathrm{pmol} / 8 \times 10^{8} \mathrm{RBCs}$. The corrected concentration for $6-\mathrm{TGN}$ ranges from 0.001 to $0.416 \mathrm{pmol} / 8 \times$ $10^{8} \mathrm{RBCs} \cdot \mathrm{mg} \cdot \mathrm{kg} \cdot \mathrm{d}$, and the median (interquartile range) concentration was $0.036(0.021,0.066) \mathrm{pmol} / 8^{*} 10^{8} \mathrm{RBC} \cdot \mathrm{mg} \cdot \mathrm{kg} \cdot \mathrm{d}$. We analyzed the concentration of 6-TGN between different variable groups. The detailed results are shown in Supplementary Table S1. In general, the levels of 6-TGN and corrected 6-TGN were not significantly different between patients with and without myelosuppression $(P=0.556$ and $P=$

Page $7 / 11$ 
0.876), and there were no significant differences between gender and AZA dose groups $(P>0.05)$. Besides, there was no obvious correlation between the levels of 6-TGN and corrected 6-TGN among different genotypes of TPMT*3C T >C, ITPA94C $>A$ and NUDT15C.415C >T $(P>$ 0.05). However, we found that the body mass index may affect the corrected 6-TGN level in this study population $(P=0.026)$.

\section{Discussion}

AZA was a classic maintenance treatment drug for AlH. However, such a drug had large individual differences and severe adverse reactions, which received widespread attention. At present, there was no clear guideline for the individualized medication of AZA in China. In this study, we found that patients with at least one genetic variant in TPMT*3C, ITPA94C $>A$ and NUDT15C.415C $>T$ were more likely to have leukopenia and neutropenia. T allele variation in NUDT15C.415C > T was an independent risk factor leading to leukopenia and neutropenia.

The blood toxicity of AZA is related to the genetic polymorphism of TPMT. Mutation or low enzyme activity leads to a high concentration of 6-TGN, which increases the risk of myelosuppression. ${ }^{6}$ Therefore, as early as 2005, the FDA had included the pre-administration TPMT genotype test in AZA's drug label. ${ }^{21}$ In AZA pharmacogenomics research, TPMT $\left({ }^{*} 2,3 A\right.$ and $\left.{ }^{*} 3 C\right)$ is to date the most widely studied single nucleotide polymorphism for AZA metabolism. The incidence is approximately $10-15 \%$ in the Caucasian population (commonly $T P M T * 3 A){ }^{22}$ However, the incidence in the Chinese population is lower than that in the Caucasian population. The literature uniformly reports that the TPMT allele (commonly TPMT*3C) accounts for less than $5 \%$ prevalence, closer to Japan and South Korea. ${ }^{23,24}$ In this study, we observed that the $C$ allele's mutation frequency in $T P M T * 3 C$ was $0.9 \%$. There are only $2(1.8 \%)$ patients with heterozygous mutations of $T P M T * 3 C$, and none of them with myelosuppression, but in TPMT*3C wild-type patients, $15(13.5 \%)$ patients suffered leukopenia, 34 (30.6\%) patients suffered thrombocytopenia, and $10(9.0 \%)$ patients suffered neutropenia. The above results indicate that $T P M T * 3 C$ gene test has limited predictive value for AZA-induced myelosuppression in the Chinese population. Therefore, although TPMT*3Chas been considered the leading risk factor for AZA-induced myelosuppression, no significant difference was observed in this study, which may be because of its low prevalence and the small sample size of this study.

ITPA polymorphism is another essential enzyme involved in the metabolism of AZA. Studies have shown that ITPA c.94C >A mutation can cause the enzyme activity to decrease, causing the toxic metabolite 6-TITP to accumulate in the body, causing flu-like symptoms, gastrointestinal reactions, skin rash, pancreatitis, and even neutropenia and liver damage, leading to interruption of treatment. ${ }^{9,25}$ The incidence of ITPA 94C > A mutations we observed in this study was $16.4 \%$, consistent with other studies. ${ }^{8}$ Only one case of homozygous mutation was observed in 113 subjects. Since he took AZA doses less than $1 \mathrm{mg} \cdot \mathrm{kg}^{-1} \cdot \mathrm{d}^{-1}$, so did not suffer myelosuppression. Although in this study, the incidences of leukopenia and thrombocytopenia in patients with heterozygous mutations were $22.9 \%(8 / 35)$ and $28.6 \%$ (10/35). However, we did not observe significant differences in the different genotypes of ITPA 94C $>A$ for AZA-induced leukopenia, thrombocytopenia and neutropenia. It may be that ITPA $94 C>A$ is mainly related to AZA-induced liver toxicity. On the other hand, the small sample size may also be the reason for the insignificant difference.

The NUDT15 belongs to the Nudix (nucleoside diphosphate linked to $x$ ) hydrolase superfamily. It mainly consists of pyrophosphohydrolase, which acts on nucleoside diphosphates linked to other moieties. The NUDT15 hydrolyzes 6-thio-GTP (TGTP) and 6-thio-GDP (TGDP) into 6thio-GMP (TGMP), which reduces their cytotoxic effects. Once NUDT15 is mutated, it will increase the cytotoxicity of mercaptopurine drugs. Most studies have shown that the incidence of NUDT15 allelic mutations in Asian populations is $8.5 \%-16 \%,{ }^{26,27}$ while it is less than $1 \%{ }^{28}$ in Caucasians. The frequency of NUDT15 mutations in IBD patients in Japan and South Korea is $12 \%$ and $10.4 \%$, respectively, but it can be as high as $32.1 \%$ in Chinese patients with autoimmune diseases. ${ }^{29}$ The frequency we observed in this study was $12.4 \%$, which is the same as the frequency of $9.4 \%$ in AlH patients reported by Xiaoli Fan et al.. ${ }^{19}$ Recent studies have found NUDT15 C. $415 C>T$ variants were associated with thiopurine-induced leukopenia, particularly in Asian populations. ${ }^{26,30-32}$ In 2014, Yang et al. ${ }^{11}$ revealed for the first time that NUDT15 c. $415 C>T$ allelic mutation is significantly associated with AZA-induced leukopenia in Korean IBD patients $\left(P=5.58 \times 10^{-43}, \mathrm{OR}=8.61\right)$. Subsequently, it was also confirmed in Japanese IBD patients that NUDT15 C.415C >T allele mutation is closely related to AZA-induced early leukopenia ${ }^{13}(P=1.92 \times 10-16, O R=28.4)$. The studies by Xiang Fei et al. ${ }^{29}$ and Xiaoli Fan et al. ${ }^{19}$ on Chinese autoimmune diseases and AlH patients also showed that NUDT15 c.415C > TSNP is significantly related to AZA-induced early leukopenia $\left(P=1.79 \times 10^{-7} ; \mathrm{OR}=7.59\right.$ and $P<0.00001 ; \mathrm{OR}=20.41$, respectively). Our result was concordant with previous studies, which showed NUDT15 C.415C $>T$ allelic mutation was associated with early leukopenia $\left(P=8.26 \times 10^{-7} ; \mathrm{OR}=7.5\right)$. At the same time, we also found that $N U D T 15 \mathrm{C} .415 \mathrm{C}>T$ mutation is implicated in AZA-induced myelosuppression with neutropenia as the primary manifestation $\left(P=3.54 \times 10^{-6} ; \mathrm{OR}=8.05\right)$. Therefore, compared with TPMT*3C and ITPA 94C >A, the detection of NUDT15 C.415C $>T$ in the Chinese population may have a better predictive value for AZA-induced myelosuppression with leukopenia and neutropenia as the primary manifestations. In addition, research has shown that NUDT15 c.415C $>T$ was associated with not only early ( $<8$ weeks) leukopenia but also middle (8-24weeks) and late ( $>24$ weeks) 
leukopenia. ${ }^{14}$ However, these findings could not be fully confirmed in our study because there was a shorter duration of follow-up (12 weeks). This happens to be the limitation of this study.

It is well known that 6-TGN is the active metabolite responsible for AZA's efficacy and cytotoxicity, and one of the side effects of AZA therapy is myelosuppression. Therapeutic drug monitoring (TDM) of the pharmacologically active metabolites of thiopurines, 6-TGN, has proven beneficial. ${ }^{33}$ However, there is no unified conclusion about the relationship between the concentration of 6-TGN in red blood cells and adverse reactions. In the research of Asada et al. ${ }^{12}$ and Xiang Fei et al. ${ }^{29}$, there was no statistically significant concentration difference observed between different NUDT15c.415C > T genotypes. However, Xiaoli Fan et al. ${ }^{19}$ reported that the 6-TGN concentration in CT genotype patients in NUDT15C.415C > T variants was significantly higher than in CC wild-type patients. The above studies have shown no significant difference in the concentration of 6-TNG between patients with or without leukopenia. This finding was replicated in our research $(P=0.556$, Table S1). Among $113 \mathrm{AlH}$ patients included in this study, we did not observe significant differences in 6-TGN concentration and Correction 6-TGN concentration among different genotypes of TPMT*3C, ITPA 94C >A and NUDT15C.415 C >T $(P>0.05$, Table S1). The same is true between different gender groups and maintenance dose groups. However, we found significant differences in the corrected concentration of 6-TGN between groups of patients with different BMI $(P=0.026$, Table S1). It indicates that obesity may be an important non-genetic factor affecting the concentration of AZA active metabolites. In recent years, studies have proposed that measuring the concentration of peripheral blood mononuclear cells (PBMC) for immunosuppressants is a new valuable biomarker to improve the therapeutic drugs monitoring. ${ }^{34}$ Combined with the results of this study, we guess that the new therapeutic monitoring method detecting the metabolites of AZA in PBMC may have more clinical value than in whole blood.

Our shortcoming is that this is a single-center study with a limited number of patients and regional limitations, which precludes adequate statistical inference. On the other hand, the follow-up time is short, and the long-term adverse reactions cannot be thoroughly evaluated. Finally, we used commercial kits to detect the most common mutations in Asian populations. The lack of comprehensive testing of AZA metabolism-related genes may lead to biased results.

\section{Conclusion}

In conclusion, our study once again confirmed that genetic variants of TPMT*3C, ITPA $94 C>A$ and NUDT15 $c .415 C>T$ are associated with AZA-induced myelosuppression in Southwest China patients with AlH. Among them, NUDT15C.415C > T variants are a potential independent risk predictor that leads to leukopenia and neutropenia. Besides, we found that the detection of 6-TGN concentration in red blood cells does not reflect AZA treatment's efficacy and toxicity. New biomarkers for AZA therapeutic drug monitoring need further research to explore.

\section{Declarations}

\section{Conflict of interest}

The authors have declared no potential conflicts of interest.

\section{Acknowledgments}

This study was supported by the Science and Technology Agency of Sichuan Province (No. 2019YFS0287).

\section{Authorship}

YB and JZ designed the research and revised the manuscript; QM wrote the manuscript; QM, LY and YZ performed the research and collected the data. QM and YL contributed to data analysis and manuscript preparation. YZ and LW helped perform the analysis with constructive discussions. All authors reviewed and approved the final manuscript.

\section{References}

1. European Association for the Study of the Liver. EASL Clinical Practice Guidelines: Autoimmune hepatitis. J Hepatol. 63, 971-1004, (2015).

2. Walker, G. J. et al. Association of Genetic Variants in NUDT15 With Thiopurine-Induced Myelosuppression in Patients With Inflammatory Bowel Disease. JAMA. 321, 773-785, (2019).

3. Qiu, Y. et al. Safety Profile of Thiopurines in Crohn Disease: Analysis of 893 Patient-Years Follow-Up in a Southern China Cohort. Medicine (Baltimore). 94, e1513; 10.1097/MD.0000000000001513 (2015). 
4. Fong, S. C., Blaker, P. A., Arenas-Hernandez, M., Marinaki, A. M. \& Sanderson, J. D. Getting the best out of thiopurine therapy: thiopurine Smethyltransferase and beyond. Biomark Med. 9, 51-65, (2015).

5. Lim, S. Z. \& Chua, E. W. Revisiting the Role of Thiopurines in Inflammatory Bowel Disease Through Pharmacogenomics and Use of Novel Methods for Therapeutic Drug Monitoring. Front Pharmacol. 9, 1107; 10.3389/fphar.2018.01107 (2018).

6. Adam de Beaumais, T. et al. Determinants of mercaptopurine toxicity in paediatric acute lymphoblastic leukemia maintenance therapy. Br J Clin Pharmacol. 71, 575-584, (2011).

7. Chouchana, L., Narjoz, C., Beaune, P., Loriot, M. A. \& Roblin, X. Review article: the benefits of pharmacogenetics for improving thiopurine therapy in inflammatory bowel disease. Aliment Pharmacol Ther. 35, 15-36, (2012).

8. Marsh, S., King, C. R., Ahluwalia, R. \& McLeod, H. L. Distribution of ITPA P32T alleles in multiple world populations. J Hum Genet. 49, 579-581, (2004).

9. Citterio-Quentin, A., Moulsma, M., Gustin, M. P., Lachaux, A. \& Boulieu, R. ITPA Activity in Children Treated by Azathioprine: Relationship to the Occurrence of Adverse Drug Reactions and Inflammatory Response. Basic Clin Pharmacol Toxicol. 122, 588-595, (2018).

10. Wahlund, M. et al. The Role of TPMT, ITPA, and NUDT15 Variants during Mercaptopurine Treatment of Swedish Pediatric Patients with Acute Lymphoblastic Leukemia. J Pediatr. 216, 150-157, (2020).

11. Yang, S. K. et al. A common missense variant in NUDT15 confers susceptibility to thiopurine-induced leukopenia. Nat Genet. 46, 10171020, (2014).

12. Asada, A. et al. NUDT15 R139C-related thiopurine leukocytopenia is mediated by 6-thioguanine nucleotide-independent mechanism in Japanese patients with inflammatory bowel disease. J Gastroenterol. 51, 22-29, (2016).

13. Kakuta, Y. et al. NUDT15 R139C causes thiopurine-induced early severe hair loss and leukopenia in Japanese patients with IBD. Pharmacogenomics J. 16, 280-285, (2016).

14. Zhu, X. et al. NUDT15 polymorphisms are better than thiopurine S-methyltransferase as predictor of risk for thiopurine-induced leukopenia in Chinese patients with Crohn's disease. Aliment Pharmacol Ther. 44, 967-975, (2016).

15. Shah, S. A., Paradkar, M., Desai, D. \& Ashavaid, T. F. Nucleoside diphosphate-linked moiety X-type motif 15 C415T variant as a predictor for thiopurine-induced toxicity in Indian patients. J Gastroenterol Hepatol. 32, 620-624, (2017).

16. Zhou, H. et al. Optimal predictor for 6-mercaptopurine intolerance in Chinese children with acute lymphoblastic leukemia: NUDT15, TPMT, or ITPA genetic variants? BMC Cancer. 18, 516; 10.1186/s12885-018-4398-2 (2018).

17. Liang, D. C. et al. NUDT15 gene polymorphism related to mercaptopurine intolerance in Taiwan Chinese children with acute lymphoblastic leukemia. Pharmacogenomics J. 16, 536-539, (2016).

18. Yang, X., Xu, H., Yang, J. \& Yang, L. Rare gene variants in a patient with azathioprine-induced lethal myelosuppression. Ann Hematol. 96, 2131-2133, (2017).

19. Fan, X., Yin, D., Men, R., Xu, H. \& Yang, L. NUDT15 Polymorphism Confer Increased Susceptibility to Thiopurine-Induced Leukopenia in Patients With Autoimmune Hepatitis and Related Cirrhosis. Front Pharmacol. 10, 346; 10.3389/fphar.2019.00346 (2019).

20. Miao, Q. et al. Highly sensitive and rapid determination of azathioprine metabolites in whole blood lysate by liquid chromatographytandem mass spectrometry. J Chromatogr B Analyt Technol Biomed Life Sci. 1136, 121802; 10.1016/j.jchromb.2019.121802 (2020).

21. Wang, L. \& Weinshilboum, R. Thiopurine S-methyltransferase pharmacogenetics: insights, challenges and future directions. Oncogene. 25, 1629-1638, (2006).

22. Khera, S. et al. Prevalence of TPMT, ITPA and NUDT 15 genetic polymorphisms and their relation to $6 \mathrm{MP}$ toxicity in north Indian children with acute lymphoblastic leukemia. Cancer Chemother Pharmacol. 83, 341-348, (2019).

23. Zhu, Q. \& Cao, Q. Thiopurine methyltransferase gene polymorphisms and activity in Chinese patients with inflammatory bowel disease treated with azathioprine. Chin Med J (Engl). 125, 3665-3670, (2012).

24. Lee, K. M. et al. Use of Thiopurines in Inflammatory Bowel Disease: A Consensus Statement by the Korean Association for the Study of Intestinal Diseases (KASID). Intest Res. 13, 193-207, (2015).

25. Ma, X. et al. Inosine triphosphate pyrophosphohydrolase (ITPA) polymorphic sequence variants in Chinese ALL children and possible association with mercaptopurine related toxicity. Int J Clin Exp Pathol. 7, 4552-4556, (2014).

26. Kakuta, Y., Kinouchi, Y. \& Shimosegawa, T. Pharmacogenetics of thiopurines for inflammatory bowel disease in East Asia: prospects for clinical application of NUDT15 genotyping. J Gastroenterol. 53, 172-180, (2018).

27. Sutiman, N. et al. Predictive role of NUDT15 variants on thiopurine-induced myelotoxicity in Asian inflammatory bowel disease patients. Pharmacogenomics. 19, 31-43, (2018). 
28. Yang, J. J. et al. Inherited NUDT15 variant is a genetic determinant of mercaptopurine intolerance in children with acute lymphoblastic leukemia. J Clin Oncol. 33, 1235-1242, (2015).

29. Fei, X. et al. NUDT15 R139C Variants Increase the Risk of Azathioprine-Induced Leukopenia in Chinese Autoimmune Patients. Front Pharmacol. 9, 460; 10.3389/fphar.2018.00460 (2018).

30. Huang, P. W., Tseng, Y. H. \& Tsai, T. F. Predictive Value of NUDT15 Variants on Neutropenia Among Han Chinese Patients with Dermatologic Diseases: A Single-Center Observational Study. Dermatol Ther (Heidelb). 10, 263-271, (2020).

31. Wang, D. S. et al. Childhood acute lymphoblastic leukemia mercaptopurine intolerance is associated with NUDT15 variants. Pediatr Res. Epub ahead of print; 10.1038/s41390-020-0868-8 (2020).

32. Kakuta, Y. et al. NUDT15 codon 139 is the best pharmacogenetic marker for predicting thiopurine-induced severe adverse events in Japanese patients with inflammatory bowel disease: a multicenter study. J Gastroenterol. 53, 1065-1078, (2018).

33. Pavlovska, K. et al. Importance of 6-Thioguanine Nucleotide Metabolite Monitoring in Inflammatory Bowel Disease Patients Treated with Azathioprine. Pril (Makedon Akad Nauk Umet Odd Med Nauki). 40, 73-79, (2019).

34. Tron, C. et al. Tacrolimus diffusion across the peripheral mononuclear blood cell membrane: impact of drug transporters. Fundam Clin Pharmacol. 33, 113-121, (2019).

\section{Supplementary Files}

This is a list of supplementary files associated with this preprint. Click to download.

- supplementarymaterials.docx

- supplementarymaterials.docx 